\title{
Direct Growth of Poly(3,4- thylenedioxythiophene) on Carbon Cloth for Supercapacitor
}

\author{
Bo Gao \\ 1. State Key Laboratory on Integrated Optoelectronics \\ College of Electronic Science and Engineering \\ Jilin University \\ Changchun, China \\ 2. Department of Resources and Environment \\ Jilin Agriculture University \\ Changchun, China \\ e-mail: gaob837@aliyun.com \\ Xin-Ping $\mathrm{He}$ \\ State Key Laboratory on Integrated Optoelectronics \\ College of Electronic Science and Engineering \\ Jilin University \\ Changchun, China \\ e-mail: 314046799 @qq.com
}

\author{
Hui Suo* \\ State Key Laboratory on Integrated Optoelectronics \\ College of Electronic Science and Engineering \\ Jilin University \\ Changchun, China \\ e-mail: suohui@jlu.edu.cn
}

\begin{abstract}
Environmentally friendly high-power energy sources are demanded increasingly nowadays due to several factors, such as global warming and climate changes, as well as the ever-growing number of portable devices and the introduction of electric vehicles. Supercapacitors as a complementary device are a kind of good candidate for energy storage due to their high power performance, long cycle life and low maintenance cost. As a kind of supercapacitor, conductive polymer such as poly(3,4ethylenedioxythiophene) has excellent properties. In this paper, through electrochemical oxidation method, 3,4ethylenedioxythiophene was polymerized on the surface of flexible carbon cloth, which can be directly used as electrode for supercapacitor. The samples were characterized by scanning electron microscopy, cyclic voltammetry and galvanostatic charge-discharge techniques. The electrochemical measurements showed such an electrode had a wide working potential window, a specific capacitance of $208.5 \mathrm{~F} \mathrm{~g}^{-1}$ and excellent cycle stability at a discharge current density of $1 \mathrm{~A} \mathrm{~g} \mathrm{~g}^{-1}$. The bendable feature of the PEDOT/CC electrodes have the potential application for flexible highpower energy storage device.
\end{abstract}

Keywords- PEDOT; carbon cloth; supercapacitor; electrode material; electrochemical oxidation

\section{INTRODUCTION}

Nowadays, there are increasing demands for environmentally friendly high-power energy sources due to several factors, such as global warming and climate changes, as well as the ever-growing number of portable devices and the introduction of electric vehicles [1]. In this sense, supercapacitors (or electrochemical capacitors) as a complementary device are a kind of good candidate for energy storage due to their high power performance, long cycle life and low maintenance cost $[2,3]$. The main electrode materials for supercapacitor include metal oxides, carbon and conductive polymers. Among them, the conductive polymer electrode materials such as polypyrrole, polyaniline or poly $(3,4-$ ethylenedioxythiophene) (PEDOT) provide flexible mechanical behavior and excellent electrochemical oxidation and reduction property [4, 5]. Especially, PEDOT has promising properties with respect to ease of synthesis, good electrical conductivity, high energy storage capacity and excellent environmental stability $[3,6]$. In recent years, many efforts have been devoted to the design and synthesis of nanostructured PEDOT electrodes [7, 8], because nanostructured electrodes are beneficial to improve the electrochemical performance of electrode materials.

Carbon materials are considered to be good candidates for nanostructural templates to support electrochemically active materials for capacitor applications [9, 10]. When deposited on high surface area carbonaceous materials, such as carbon nanotubes [11], carbon fibers [12], some polymeric capacitors exhibit enhanced performance, which is due to the fact that the carbon substrate provides a higher mechanical stability for the polymer films, and absorbs the mechanical stress in the polymer caused by the insertion/deinsertion of counter ions during the cycling 
process. However, these nanocomposites are in a way limited in their scalability. The flexible carbon cloth (CC) sheets not only have a high specific surface area but also have an excellent 3D conductive skeleton, which support a high electrolytic accessible surface area of electrochemical active materials and a direct path for electrons. Here, the direct-grown PEDOT modified CC sheet was obtained by electrochemical oxidation synthesis, and moreover, the bendable feature of the PEDOT/CC electrodes also have the potential application for flexible high-power energy storage device.

\section{EXPERIMENTAL}

A commercially available carbon cloth (CC) (Jilin Shuang Ya carbon material factory) was used as the supporting material for PEDOT. For control experiment, a CC sheet with dimensions of $10 \mathrm{~mm} \times 10 \mathrm{~mm} \times 1.0 \mathrm{~mm}$ was treated in $25 \%$ hydrochloric acid at $40^{\circ} \mathrm{C}$ for $30 \mathrm{~min}$, cleaned successively in acetone and deionized water until neutral and dried at $50^{\circ} \mathrm{C}$ for $5 \mathrm{~h}$. Other chemicals used for PEDOT growth, viz. 3,4-ethylenedioxythiophene (EDOT) monomer (Herochem, 99.9\%), $\mathrm{H} 2 \mathrm{SO} 4, \mathrm{HCl}, \mathrm{LiClO} 4$ were of analytical-reagent grade and used without any further treatment.

To prepare the electrode sheet of PEDOT/CC material, the electrochemical oxidation of EDOT $(15 \mathrm{mM})$ was performed in $\mathrm{LiClO}(0.1 \mathrm{M})$ aqueous solution. The potentiostatic mode with constant potential of $0.9 \mathrm{~V}$ (vs. $\mathrm{Ag} / \mathrm{AgCl} \mathrm{RE}$ ) for 10, 20, 30, 40 and 50 min was applied to synthesize the uniform coverage of PEDOT thin film on $\mathrm{CC}$, and then the as-grown samples rinsed with distilled water and dried at $50^{\circ} \mathrm{C}$ under vacuum for $3 \mathrm{~h}$.

The morphology of the PEDOT/CC was characterized by a field-emission scanning electron microscopy (FESEM, JSM-6700F, JEOL, Japan) at 5kV. Electrochemical experiments were carried out in a three-electrode system with a CHI 660D electrochemical workstation with $\mathrm{PEDOT} / \mathrm{CC} \quad(10 \mathrm{~mm} \times 10 \mathrm{~mm})$ as working electrode, platinum wire as auxiliary electrode and $\mathrm{Ag} / \mathrm{AgCl}(3 \mathrm{M}$ $\mathrm{KCl}$ ) as reference electrode. $0.5 \mathrm{M} \mathrm{H} 2 \mathrm{SO} 4$ was used as electrolyte. Cyclic voltammetry (CV) was carried out in a potential range of $-0.4 \sim 0.8 \mathrm{~V}$ at varying scan rates. Galvanostatic charge/discharge (GCD) curves were demonstrated at step increasing current densities with the cutoff voltage of $0.8 \mathrm{~V}$. The battery testing system (Neware) was used to measure the cycle life of the sample with the cycle times of 1000 . In order to eliminate $\mathrm{O} 2$ in the electrolyte, the $\mathrm{H} 2 \mathrm{SO} 4$ solution was charged pure N2 for $5 \mathrm{~min}$ before the electrochemical measurements.

\section{RESULTS AND DISCUSSION}

\section{A. Morphology and structure}

The SEM images of the CC modified before and after are shown in Fig .1, inset of which are the magnification of the corresponding SEM images. Fig .1 a shows the fiber of blank CC, which has a smooth surface with some fine grooves. These fibers intertwine with each other, forming a 3D highly porous structure, which is in favor of the transport of electrons and ions in the supercapacitor devices. After modification with PEDOT (electrodeposition of EDOT for 40min), the fibers of CC sheet are coated with a rough layer, as shown in Fig .1b. There are some particles with spherical shape protruding from the rough layer. From inset of Fig .1b, we can see the microstructure of PEDOT film, seeming as many disks assembled together orderly with many grooves between them.

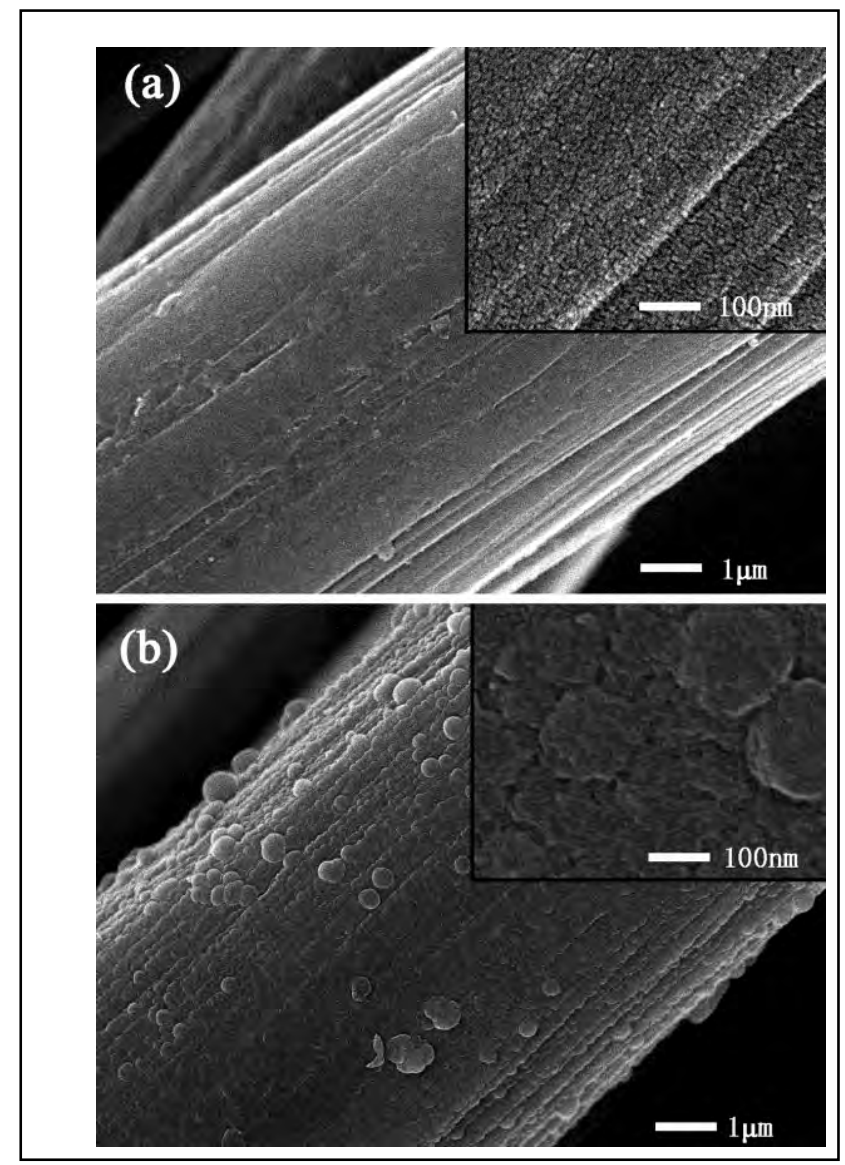

Figure 1. SEM of (a) blank CC and (b) PEDOT/CC electrode under constant potential of $0.9 \mathrm{~V}$ for $40 \mathrm{~min}$.

\section{B. Electrochemical property}

The electrochemical performance of the PEDOT/CC was tested via $\mathrm{CV}$ and the GCD experiments through a three-electrode system. Fig .2 shows CV curves of blank $\mathrm{CC}$, PEDOT/CC polymerized for different period of time in $0.5 \mathrm{M} \mathrm{H} 2 \mathrm{SO} 4$ electrolyte at a scan rate of $25 \mathrm{mVs}-1$. The CV curve of CC sheet has no pair of redox peaks, which exhibits typical double-layer capacitance characteristics. For the PEDOT/CC, several quite symmetrical CV curves can be observed within the potential window between -0.4$0.8 \mathrm{~V}$, which implies the capacitive and reversible characteristics of PEDOT/CC electrodes. It is commonly believed that the electrochemical capacitance is proportional the area under the CV curve. From Fig .2, we can see that the area of $\mathrm{CV}$ curves are larger than that of blank CC, and increases obviously with the period of polymerization time from curve 2 to curve 5 , indicating the growth of PEDOT film on CC surface. However, the area under $\mathrm{CV}$ curve 6 increased a little compared with that of curve 5, which implies that the extension of polymerization time has little effect for the increase of electrochemical capacitance after the polymerization time reached 40min. Therefore, we selected the sample with polymerization time $40 \mathrm{~min}$ for the next experiment. 


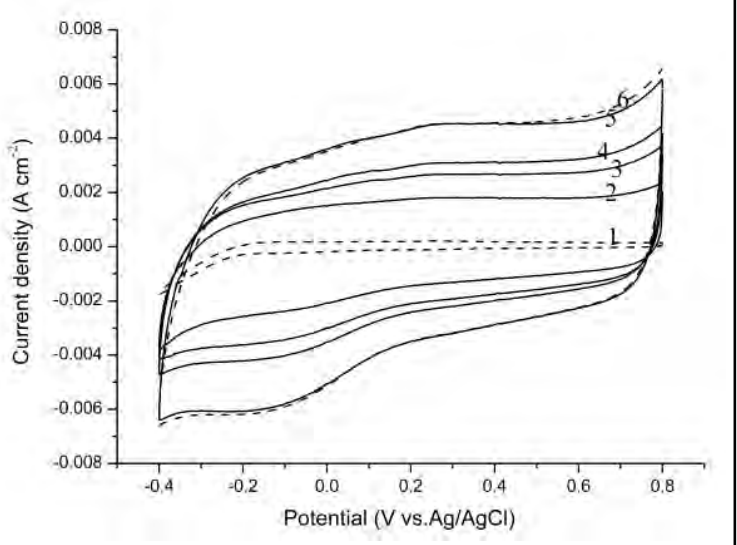

Figure 2. CV curves of blank (1) $\mathrm{CC}$ and PEDOT/CC electrode under constant potential of $0.9 \mathrm{~V}$ for (2) $10 \mathrm{~min}$, (3) $20 \mathrm{~min}$, (4) $30 \mathrm{~min}$, (5) $40 \mathrm{~min}$ and (6) $50 \mathrm{~min}$.

Fig .3 shows the CV curves of PEDOT/CC with polymerization time $40 \mathrm{~min}$ at various scan rates of 25,50 , $75,100 \mathrm{mV} \mathrm{s}-1$. The current density increases with the scan rates and the shape of the $\mathrm{CV}$ curves also changes accordingly. The scan rate plays an important role in the diffusion of electrolyte ions into the exterior material of electrode during potential cycling. At higher scan rates, the working ions can only approach the external CC electrode, which result in the fact that the internal material on the electrode does not respond actively for contributing to the pseudo-capacitance.

Fig .4 displays the GCD curves of PEDOT/CC electrode with polymerization time $40 \mathrm{~min}$ at different current densities of $1,2,5,10$ A g-1. The GCD curves behave mirror-like shape, indicating a good supercapacitor property. The specific capacitance $(\mathrm{Cm})$ can be calculated with (1) [13] below:

$$
\mathrm{C}_{\mathrm{m}}=\frac{\mathrm{C}}{m}-\frac{I \Delta t}{\Delta V m}(1)
$$

Where $\mathrm{Cm}$ is the specific capacitance in $\mathrm{F}$ g- 1 , I is the charge-discharge current in $\mathrm{A}, \mathrm{t}$ is the discharge time in $\mathrm{s}$, $\Delta \mathrm{V}$ is potential drop during discharge in $\mathrm{V}$ and $\mathrm{m}$ is the mass of active material in $\mathrm{g}$. According to the above equation, the specific capacitance of PEDOT/CC is calculated as 208.5, 180.2, 155.5, $147 \mathrm{~F}$ g-1at 1, 2, 5, $10 \mathrm{~A}$ $\mathrm{g}-1$, respectively. A conclusion can be reached that the specific capacitance decreased as the discharge current density increasing for the PEDOT/CC electrode.

An important factor for the PEDOT/CC electrode is the cycle stability subjected to long-term charge-discharge cycles. Fig .5 shows the specific capacitance of $\mathrm{PEDOT} / \mathrm{CC}$ at a discharge current density of $1 \mathrm{~A}$ g-1 in $0.5 \mathrm{M} \mathrm{H} 2 \mathrm{SO} 4$ electrolyte for 1000 cycles. PEDOT/CC electrode exhibits good cycling behavior. Compared with the initial specific capacitance value $208.5 \mathrm{~F}$ g-1, it decreased to $188 \mathrm{~F} \mathrm{g-1}$ in the first 300 cycles and kept nearly the same in the subsequent 700 cycles. The initial $10.05 \%$ decline of specific capacitance may be due to the loss of unstable PEDOT particles during the intercalating/deintercalating process of electron and electrolyte ions.

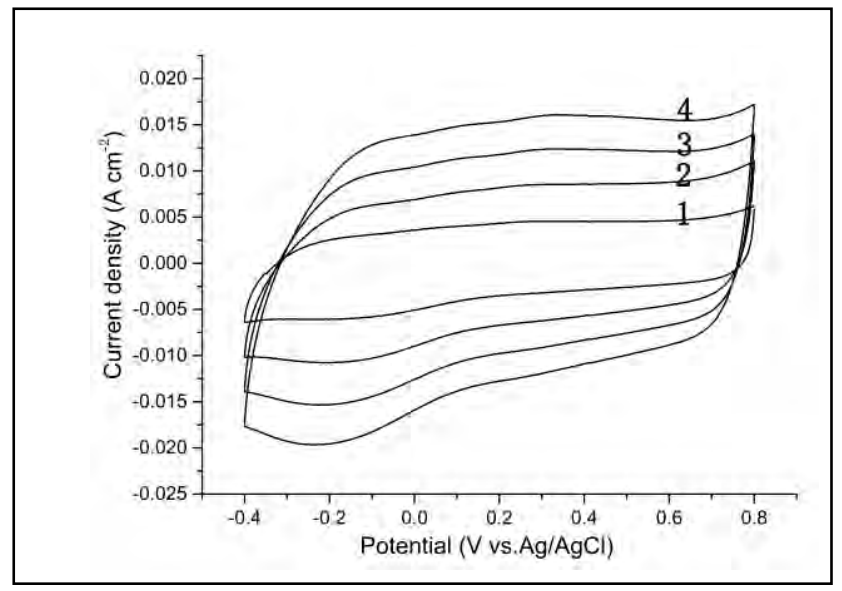

Figure 3. CV curves of PEDOT/CC with polymerization time $40 \mathrm{~min}$ at various scan rates of (1) 25 , (2) 50 , (3) 75 , (4) $100 \mathrm{mV} \mathrm{s}^{-1}$.

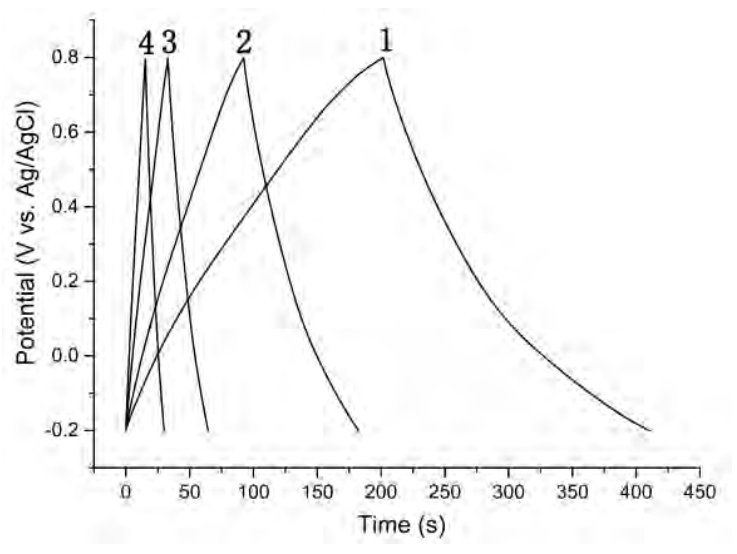

Figure 4. GCD curves of PEDOT/CC electrode with polymerization time $40 \mathrm{~min}$ at different current densities of (1)1, (2) 2, (3) 5, (4) $10 \mathrm{~A} \mathrm{~g}^{-1}$.

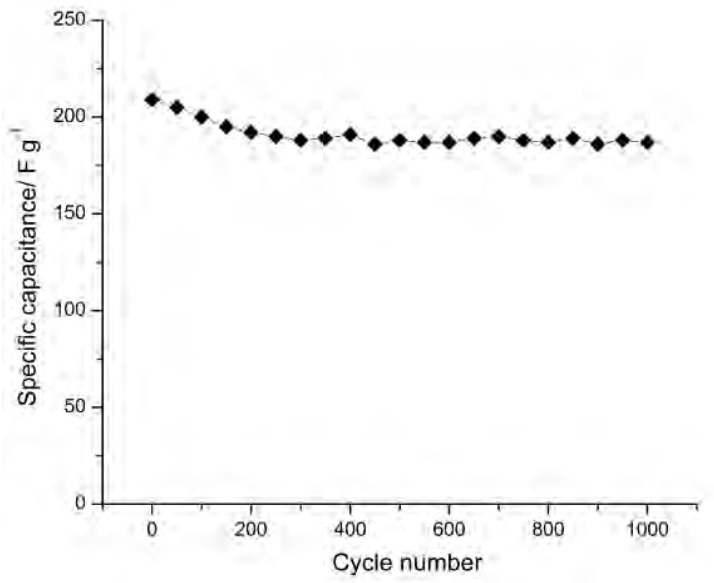

Figure 5. Variation of specific capacitance as a function of cycle number for the PEDOT/CC electrode in the $0.5 \mathrm{M} \mathrm{H}_{2} \mathrm{SO}_{4}$ electrolyte at a discharge current density of $1 \mathrm{~A} \mathrm{~g} \mathrm{~g}^{-1}$. 


\section{CONCLUSIONS}

In summary, the direct-grown of PEDOT on the CC electrode was successfully synthesized via electrochemical polymerization. Disk-like nanostructure of PEDOT assembled together orderly with many grooves between them was observed by SEM. The PEDOT/CC electrode exhibits wide operated potential window of $1.2 \mathrm{~V}$ in the electrolyte of $\mathrm{H} 2 \mathrm{SO} 4$ and achieves specific capacitance of $208.5 \mathrm{~F} \mathrm{g-1}$ at a discharge current density of $1 \mathrm{~A} \mathrm{g-1.} \mathrm{Such}$ an electrode also exhibits good electrochemical cycle stability with about $90 \%$ initial specific capacitance remained after 1000 continuous cycles. Obviously, the electrochemical oxidation fabrication and excellent electrochemical performance provide great potential for this type of electrode for supercapacitors.

\section{ACKNOWLEDGMENT}

This work was supported by State Key Laboratory on Integrated Optoelectronics, College of Electronic Science and Engineering, Natural Science Foundation of P. R. China (Item No. 61374218).

\section{REFERENCES}

[1] L. L. Zhang, X. S. Zhao, "Carbon-based materials as supercapacitor electrodes," [J]. Chemical Society Review, 2009, 38, 2520-2531.

[2] M. Fabretto, K. Zuber, C. Hall, P. Murphy, H. J. Griesser, "The role of water in the synthesis and performance of vapour phase polymerized PEDOT electrochromic devices," [J]. J. Mater. Chem. 2009, 19 (42), 7871-7878.

[3] A. Laforgue, "All-textile flexible supercapacitors using electrospun poly(3,4- ethylenedioxythiophene) nanofibers," [J]. J. Power Source, 2011, 196, 559-564.
[4] H. Zhou, H. Chen, S. Luo, G. Lu, W. Wei, Y. Kuang, "The effect of the polyaniline morphology on the performance of polyaniline supercapacitors," [J]. J. Solid State Electrochem 2005, 9 (8), 574580 .

[5] W. Sun, X. Chen, "Preparation and characterization of polypyrrole films for three-dimensional micro supercapacitor," [J]. J. Power Source 2009, 193 (2), 924-929.

[6] J. A. Lee, M. K. Shin, S. H. Kim, S. J. Kim, G. M. Spinks, G. G. Wallace, R. Ovalle-Robles, M. D. Lima, M. E. Kozlov, R. H. Baughman, "Hybrid Nanomembranes for High Power and High Energy Density Supercapacitors and Their Yarn Application," [J]. ACS Nano, 2012, 6 (1), 327-334.

[7] C. Zhu, J. Zhai, D. Wen, S. Dong, "Graphene oxide/polypyrrole nanocomposites: one-step electrochemical doping, coating and synergistic effect for energy storage," [J]. J. Mater. Chem., 2012, 22, 6300-6306.

[8] T. Abdiryin, A. Ubul, R. Janal, F. Xu, A. Rahman, "Electrochemical properties of the poly(3,4-ethylenedioxythiophene)/single-walled carbon nanotubes composite synthesized by solid-state heating method," [J]. Synth. Metasl, 2012, 162 (17-18), 1604-1608.

[9] Y. G. Wang, H. Q. Li, Y. Y. Xia, "Ordered Whiskerlike Polyaniline Grown on the Surface of Mesoporous Carbon and Its Electrochemical Capacitance Performance," [J]. Adv. Mater, 2006, 18 (19), 2619-2623.

[10]D. Zhang, X. Zhang, Y. Chen, P. Yu, C. Wang, Y. Ma, "Enhanced capacitance and rate capability of graphene/polypyrrole composite as electrode material for supercapacitors," [J]. J. Power Sources, 2011, 196 (14), 5990-5996.

[11] Z. L. Wang, R. Guo, L. X. Ding, Y. X. Tong, G. R. Li, "Controllable Template-Assisted Electrodeposition of Single- and Multi-Walled Nanotube Arrays for Electrochemical Energy Storage," [J]. Sci. Rep., 2013, 3, 1204.

[12] H. R. Ghenaatian, M. F. Mousavi, M. S. Rahmanifar, "High performance hybrid supercapacitor based on two nanostructured conducting polymers: Self-doped polyaniline and polypyrrole nanofibers" [J]. Electrochim. Acta, 2012, 78 (1), 212-222.

[13] A. K. Mishra, S. Ramaprabhu, "Functionalized Graphene-Based Nanocomposites for Supercapacitor Application," [J]. J. Phys. Chem. C 2011, 115, 14006-14013 